\title{
Tra scienza e professione
}

\author{
Marco Petitta \\ Presidente del Comitato Italiano IAH \\ Marco.Petitta@uniroma1.it
}

Il nuovo corso di Acque Sotterranee, da sempre rivista di riferimento per chi si occupa di idrogeologia e gestione delle risorse idriche sotterranee, ha inteso coinvolgere in questa iniziativa anche il Comitato Italiano della Associazione Internazionale Idrogeologi (IAH), rinnovato all'inizio di quest'anno.

Pertanto, da questo numero i lettori troveranno questa rubrica curata appunto dal Comitato Italiano IAH, che intende rivolgersi non soltanto al mondo accademico e scientifico, ma ancor di più vuole coinvolgere gli aspetti professionali dell'idrogeologia, con particolare attenzione alle aspettative dei professionisti del settore.

In questo ambito, di volta in volta proporremo temi di discussione e di riflessione, a partire da eventi, convegni e congressi che riguardino l'idrogeologia.

Confidiamo che la collaborazione con Acque Sotterranee vada ben oltre queste pagine, tramite iniziative congiunte in via di definizione.

E' opportuno innanzitutto presentare il nuovo Comitato Italiano, che oltre ai ricercatori presenti nella foto, comprende il precedente Presidente e rappresentante della Societa' Geologica Italiana Prof. Giovanni Barrocu e tre soci esponenti del mondo professionale, recentemente cooptati: Paolo Cerutti, Antonio De Matteis e Andrea Sottani. Le attività del Comitato si sono concentrate in questi primi mesi nella riorganizzazione delle rappresentanze presso le Commissioni e i Network di studio a livello internazionale, nonché nel patrocinio del Convegno Flowpath 2012, organizzato dall'Università di Bologna nel mese di giugno. Sono stati inoltre intrapresi contatti con le altre associazioni tecnico-scientifiche del settore e soprattutto con il Consiglio Nazionale dei Geologi, per concordare con quest'ultimo una proposta di aggiornamento professionale esplicitamente dedicata agli aspetti idrogeologici, che intendiamo promuovere entro fine anno. Inoltre, è stato lanciato il nuovo sito web dell'Associazione, consultabile alla pagina www.iahitaly.it.

Le attività della IAH includono ovviamente anche la possibilità di partecipare a convegni e congressi internazionali, dove si ha l'occasione di aggiornarsi in merito alle ultime novità e tendenze su temi specifici. Ad esempio, nello scorso mese di maggio, si è svolta a Praga la conferenza internazionale dedicata alla circolazione idrica in rocce fratturate (Groundwater in Fractured Rocks), durante la quale si sono affrontati molti temi di interesse non soltanto scientifico, ma anche tecnico, grazie alla presenza di professionisti del settore e anche di società pubbliche e private.

Le prime sessioni della conferenza sono state dedicate alla struttura idrogeologica e al flusso in rocce fratturate, con esposizione di casi di studio comprendenti la caratterizzazione geomeccanica e idraulica delle fratture. Sono stati presentati metodi per la determinazione della conducibilità idraulica, anche tramite interpretazione di prove di emungimento appositamente realizzate, affiancate da prove di permeabilità con packer su tratti limitati degli acquiferi in esame, e da prove tramite mulinelli da pozzo (flowmeter), oltre che da indagini geofisiche. Particolare attenzione è stata riservata ai rilevamenti di terreno, in grado di evidenziare le differenze di permeabilità dovute ad alterazione o a fratturazione di superficie connessa ai litotipi e alla tettonica. Anche alcuni esempi a scala di laboratorio hanno indagato la problematica della valutazione della permeabilità in ammassi fratturati, mentre alla scala opposta sono state illustrate le potenzialità delle immagini da satellite o da foto aerea. Altre presentazioni hanno trattato delle indagini in galleria o comunque nel sottosuolo. In generale, si è posto l'accento sul ruolo sia idrostatico che idrodinamico delle fratture, ribadendo che la permeabilità di un ammasso roccioso fratturato va determinata sommando, con vari metodi, la conducibilità idraulica della roccia a quella delle fratture, quest'ultima quasi sempre determinante nel condizionare il flusso idrico sotterraneo.

Altro argomento oggetto di un numero limitato di interventi è stato quello sulle caratteristiche chimico-fisiche ed isotopiche delle acque sotterranee in acquiferi fratturati, durante i quali si è posto l'accento sulla solubilità e sulle possibilità offerte dai traccianti naturali e artificiali.

Un tema particolarmente indirizzato agli aspetti tecnici è stato quello della prospezione e perforazione di pozzi in acquiferi fratturati, tramite esposizione di varie tecniche e metodiche di realizzazione dei pozzi, sia per ottimizzarne il prelievo e l'efficienza che per coniugare i dati idrodinamici con quelli geotecnici provenienti da perforazioni in rocce fratturate.

Altro tema tecnico e' stato sviscerato dagli interventi sui metodi di investigazione e relative interpretazioni in mezzi fratturati, comprendenti geofisica in foro, prove di emungimento di vario genere, approcci integrati dalla perforazione ai logs interpretativi, packer test e prove con traccianti.

I risultati presentati hanno ribadito la possibilità di utilizzare diverse tecniche per la caratterizzazione del flusso in acquiferi fratturati, con diversi spunti innovativi sia metodologici che applicativi.

Lo studio delle caratteristiche delle fratture e della loro influenza sulla circolazione idrica sotterranea è stato oggetto di un'apposita sessione, durante la quale hanno preso la parola due autentici specialisti del settore, gli statunitensi John Sharp e Shlobo Neuman, che attraverso i loro interventi ad invito 
hanno fatto il punto sia sulle basi teoriche che su esperimenti di laboratorio finalizzati alla valutazione della permeabilità delle fratture.

Un'ampia sessione di modellistica idrogeologica ha evidenziato le notevoli potenzialità dei modelli matematici, applicabili sia alla scala di sito che a scala di estremo dettaglio o anche regionale, sempre a condizione di avere a disposizione set di dati abbastanza dettagliati ed attendibili da consentire l'allestimento di un modello rappresentativo della realtà di terreno. Gli approcci seguiti comprendono il poroso equivalente, i modelli con discretizzazione delle fratture (discrete fracture network) come quelli a doppia porosità. Interessanti sviluppi presenta la possibilità di adottare modelli di trasporto in soluzione, utilizzabili per ricostruire i percorsi dei contaminanti o semplicemente dei soli ioni disciolti. Problematica particolarmente sentita è anche quella della scala di modellazione in funzione della scala di indagine e di intervento, che nel campo delle rocce fratturate influenza notevolmente gli stessi valori di conducibilità idraulica misurati e modellati.

Infine, la sessione conclusiva ha incluso casi di studio relativi alla protezione della risorsa in acquiferi fratturati, tramite esempi di delimitazione delle aree di salvaguardia e spunti riguardanti i temi ecologici e di interconnessione tra diversi livelli acquiferi, dove i pozzi possono svolgere il ruolo di fonte di contaminazione.

In definitiva, dalla Conferenza sono emersi molti spunti interessanti e aggiornamenti fondamentali sul tema della circolazione in ammassi fratturati, che se da un lato hanno ribadito gli approcci ormai classici e le metodiche di indagine adottabili in questi studi, dall'altro hanno posto l'attenzione sui recenti sviluppi metodologici e tecnologici, soprattutto nel settore delle indagini in foro e delle relative interpretazioni anche modellistiche. Aspetti questi di sicuro interesse sia tecnico che scientifico per gli idrogeologi del nostro Paese, dove la casistica degli acquiferi fratturati è ampia come tipologie e diffusa sul territorio, dai rilievi montuosi alle aree costiere.

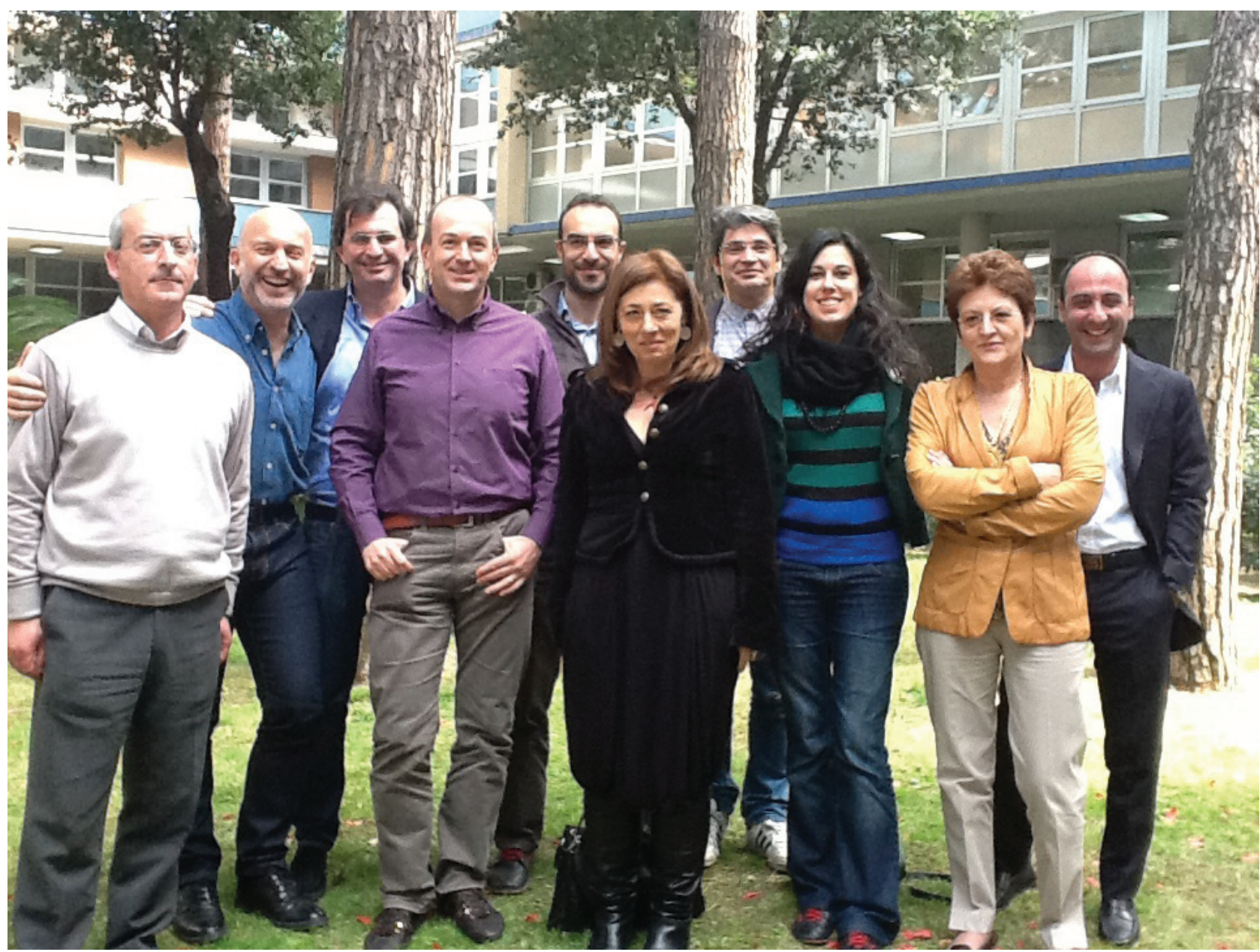

Fig. 1 - I membri del Comitato Italiano IAH Da sinistra: Vincenzo Piscopo, Alessandro Gargini, Maurizio Polemio, Marco Petitta, Marco Masetti, Daniela Ducci, Paolo Fabbri, Viviana Re, Dolores Fidelibus, Fulvio Celico 